\title{
Properties of Linguistic 2-tuple Judgment Matrix with Additive Consistency
}

\author{
Xixiang Zhang ${ }^{1,2}$ Jing Lei $^{1,3}$ Bao-an Yang ${ }^{1}$ \\ ${ }^{1}$ Glorious Sun School of Business and Management, Donghua University, Shanghai 200051, P.R.China \\ ${ }^{2}$ Information Engineering School, Jiaxing College, Jiaxing 314001, P.R .China \\ ${ }^{3}$ Shanghai Business School, Shanghai 200235, P.R China
}

\begin{abstract}
Many scholars focus on linguistic 2-tuple to solve group decision making problem based on linguistic information because it can avoid information losing in the process of manipulating and computing linguistic information. However, there are few researches on the properties of linguistic 2-tuple judgment matrix with additive consistency till now. This paper studies the additive consistency of linguistic 2-tuple judgment matrix and puts forward several propositions about linguistic 2-tuple judgment matrix with additive consistency. These propositions can provide methods to judge additive consistency of linguistic 2-tuple judgment matrix and to build linguistic 2-tuple judgment matrix with additive consistency.
\end{abstract}

Keywords: Linguistic 2-tuple, Linguistic 2-tuple judgment matrix, Additive consistency

\section{Introduction}

In real world, the uncertainty, constraints, and even unclear knowledge of the experts imply that decision makers cannot provide exact number to express their preferences. The use of linguistic labels makes expert judgment more reliable and consistent [1]-[2]. We can find different linguistic computational models for aggregating linguistic preference variables: the first model is the approximate computational model based on the extension principle, which uses fuzzy numbers to represent linguistic labels [3]-[4]; the second model is the ordinal linguistic computational model, which makes direct computations on labels [5]-[6]; the third model is the 2-tuple linguistic computational model, which uses the 2-tuple linguistic presentation model and makes linguistic computations [7]-[8]. The 2-tuple linguistic presentation model can avoid information loss in processing and computing linguistic information, and maintain accuracy and consistency of linguistic information [8]. Therefore, many scholars are focusing on group decision making based on linguistic 2-tuple. Herrera and Martinez (2001) introduces the linguistic hierarchical structure and transformation functions among different levels of a linguistic hierarchy, and propose the multi-expert decision making model based linguistic 2-tuples to deal with multigranular hierarchical linguistic contexts [7]. Delgado, Herrera and Herera-Videma et al present a distributed intelligent agent model where the communication of the evaluation of information among the agents is carried out by using 2-tuple linguistic representation model to endow the retrieval process [9]. Wang and Fan (2003) propose a group decision making method using linguistic 2-tuple to deal with linguistic information [10]. Li and Fan (2003) define linguistic ordered weighted averaging (LOWA) operator and Linguistic implication function of linguistic 2-tuple and put forward a model for group decision making [11]. Yu and Fan (2006) propose a new maximal tree clustering analysis method multiple attribute clustering analysis problems with linguistic assessment information, which manipulates linguistic 2-tuple and clusters the alternatives [12]. Liao, Li and Dong (2006) put forward a linar programming model, which firstly use the 2-tuple linguistic representation model to aggregate the linguistic information and compute group consistency and group inconsistency based on the idea of LINMAP, and then build the ideal solution and the weights of experts [13]. Jiang and Xing ( 2007 ) define linguistic 2-tuple judgment matrix, additive consistency and satisfy consistency of linguistic 2-tuple judgment matrix, and find some methods to judge the additive consistency and satisfied consistency of linguistic 2-tuple judgment matrix [14].

Researches on consistency analysis of judgment matrix with fuzzy linguistic information are further studied [15]-[17]. However, few researches focus on properties of linguistic 2-tuple [16]. This paper studies properties of the linguistic 2-tuple judgment matrix which is additive consistent and propose several propositions. The rest structure of this paper is as follows: Part 2 discusses the 2-tuple linguistic representation model and its operators; Part 3 defines the linguistic 2-tuple judgment matrix and its additive consistency; Part 4 proposes several propositions about linguistic 2-tupole judgment matrix with 
additive consistency and prove them; Part 5 gives the conclusion.

\section{Linguistic 2-tuple and its operators}

Spanish professor Francisco Herrera uses the 2-tuple linguistic representation model to manipulate linguistic information [5]. This model has the following advantages for representing the linguistic information over classical model [8]: the linguistic domain can be treated as continuous; the linguistic computational model based on linguistic 2-tuple carries out processes of computing with words more easily and without loss of information; the result of the processes of computing with words are always expressed in the initial linguistic domain.

The linguistic 2-tuple representation model takes as a basis the symbolic model and symbolic translation to represent the linguistic information using a pair of values called linguistic 2-tuple (written as $\left(s_{i}, \alpha\right)$, $S_{i}$ is a linguistic term and $\alpha$ is a numeric value ).

\subsection{Linguistic 2-tuple}

Suppose $S=\left\{s_{0}, s_{1}, \cdots, s_{g}\right\}$ be a set of labels assessed in a linguistic term set with odd elements, which has the following properties: (1)ordered: when the index i $\geqslant \mathrm{j}$, there must exist $\mathrm{s}_{\mathrm{i}} \geqslant \mathrm{s}_{\mathrm{j}}$; (2)a negation operator: $\operatorname{Neg}\left(s_{i}\right)=s_{g-i}$; (3) there exists a min and max operator: $s_{i}$ $\geqslant \mathrm{s}_{\mathrm{j}}$ means $\max \left(\mathrm{s}_{\mathrm{i}}, \mathrm{s}_{\mathrm{j}}\right)=\mathrm{s}_{\mathrm{i}}$ and $\min \left(\mathrm{s}_{\mathrm{i}}, \mathrm{s}_{\mathrm{j}}\right)=\mathrm{s}_{\mathrm{j}}[16]$.

Definition 1 [9] Let $\beta$ be the result of an aggregation of the indexes of a set $S=\left\{s_{0}, s_{1}, \cdots, s_{g}\right\}$, for example, the result of a symbolic aggregation operation. $\beta \in[0, g]$ and $g+1$ is the cardinality of $\mathrm{S}$. Let $i=\operatorname{round}(\beta)$ and $\alpha=\beta-i$ be two values, such that, $i \in[0, g]$ and $\alpha \in[-0.5,0.5]$ then $\alpha$ is called a Symbolic Translation.

Symbolic Translation is a numberic value between $[-0.5,0.5]$, which shows the difference between $\beta$ that is the aggregation result of elements in $\mathrm{S}$ and the closest element $\mathrm{s}_{\mathrm{i}} \mathrm{S}$.

Definition 2 [9] Let $S=\left\{s_{0}, s_{1}, \cdots, s_{g}\right\}$ be a linguistic term set and $\beta \in[0, g]$ be a value representing the result of a symbolic aggregation operation, then the 2-tuple that expresses the equivalent information to $\beta$ is obtained with the following function:

$$
\nabla:[0, g] \rightarrow S \times[-0.5,0.5]
$$

$\nabla(\beta)=\left(s_{i}, \alpha\right)$, with $\left\{\begin{array}{c}\mathrm{s}_{\mathrm{i}}, i=\operatorname{round}(\beta) \\ \alpha=\beta-i, \alpha \in[-0.5,0.5]\end{array}\right.$

Where round(.) is the usual round operation, $\mathrm{s}_{\mathrm{i}}$ had the closest index label to $\beta$.

Proposition 1 [9] Let $S=\left\{s_{0}, s_{1}, \cdots, s_{g}\right\}$ be a linguistic term set and $\left(s_{i}, \alpha\right)$ be a 2-tuple. There is always a $\nabla^{-1}$ function, such that, from a 2-tuple it returns its equivalent numerical value $\beta \in[0, g]$, which is:

$$
\begin{gathered}
\nabla^{-1}: S \times[-0.5,0.5] \rightarrow[0, g] \\
\nabla^{-1}\left(s_{i}, \alpha\right)=i+\alpha=\beta
\end{gathered}
$$

From definition 1 , definition2 and proposition 1 , we can conclude that the conversion of a linguistic term into a linguistic 2-tuple consist of adding a value 0 as the symbolic translation, which is :

$$
\theta\left(s_{i}\right)=\left(s_{i}, 0\right)
$$

\subsection{Operators of linguistic 2-tuple}

Operation model of linguistic 2-tuple can be obtained according to the linguistic 2-tuple representation model, such as, the negation operator of the linguistic 2-tuple, the comparing operator of the linguistic 2tuple, the aggregation operators of the linguistic 2tuple.

(1) A linguistic 2-tuple negation operator [8]

$$
\operatorname{Neg}\left(\left(s_{i}, \alpha\right)\right)=\nabla\left(g-\left(\nabla^{-1}\left(s_{i}, \alpha\right)\right)\right)
$$

(2) Linguistic 2-tuple comparison operators [9]

Let $\left(s_{i}, \alpha\right)$ and $\left(s_{i}, \beta\right)$ be two linguistic 2tuples, then:

If $\mathrm{i}<\mathrm{j}$, then $\left(s_{i}, \alpha\right)$ is smaller than $\left(s_{j}, \beta\right)$, noted as $\left(s_{i}, \alpha\right)<\left(s_{i}, \beta\right)$;

If $\mathrm{i}=\mathrm{j}$, then

if $\alpha>\beta$ then $\left(s_{i}, \alpha\right)$ is bigger than $\left(s_{i}, \beta\right)$, noted as $\left(s_{i}, \alpha\right)>\left(s_{i}, \beta\right)$;

if $\alpha=\beta$ then $\left(s_{i}, \alpha\right),\left(s_{i}, \beta\right)$ represents the same linguistic information, noted as $\left(s_{i}, \alpha\right)=\left(s_{i}, \beta\right)$;

if $\alpha<\beta$ then $\left(s_{i}, \alpha\right)$ is smaller than $\left(s_{i}, \beta\right)$, noted as $\left(s_{i}, \alpha\right)<\left(s_{i}, \beta\right)$.

(3) Linguistic 2-tuple aggregation operators

Definition 3 [13] Let $\left(s_{1}, \alpha_{1}\right),\left(s_{1}, \alpha_{2}\right), \cdots,\left(s_{n}, \alpha_{n}\right)$ be a set with $\mathrm{n}$ linguistic 2-tuples, the average operator of linguistic 2tuples $\xi$ is: 


$$
\begin{aligned}
& \xi\left(\left(s_{1}, \alpha_{1}\right),\left(s_{2}, \alpha_{2}\right), \cdots,\left(s_{n}, \alpha_{n}\right)\right)=(\bar{s}, \bar{\alpha}) \\
& \quad=\nabla\left(\frac{1}{n} \sum_{i=1}^{n} \nabla^{-1}\left(s_{i}, \alpha_{i}\right)\right)
\end{aligned}
$$

Definition

Let

$\left(s_{1}, \alpha_{1}\right),\left(s_{1}, \alpha_{2}\right), \cdots,\left(s_{n}, \alpha_{n}\right)$ be a set with $\mathrm{n}$ linguistic 2-tuples and $\omega=\left(\omega_{1}, \omega_{2}, \cdots, \omega_{n}\right)$ be the related weighted vector with $\sum_{i=1}^{n} \omega_{i}=1$, then the weighted average operator of linguistic 2-tuples $\xi^{\omega}$ is

$$
\begin{gathered}
\xi^{\omega}\left(\left(s_{1}, \alpha_{1}\right),\left(s_{2}, \alpha_{2}\right), \cdots,\left(s_{n}, \alpha_{n}\right)\right)=(\hat{s}, \hat{\alpha}) \\
=\nabla\left(\frac{\sum_{i=1}^{n} \nabla^{-1}\left(s_{i}, \alpha_{i}\right) \omega_{i}}{\sum_{i=1}^{n} \omega_{i}}\right)
\end{gathered}
$$

\section{Linguistic 2-tuple judgment matrix and its additive consistency}

Let $\mathrm{S}=\left\{\mathrm{s}_{0}, \mathrm{~s}_{1}, \cdots, \mathrm{s}_{\mathrm{g}}\right\}$ be a natural language term set and $X=\left\{x_{1}, x_{2}, \cdots, x_{n}\right\}$ be the set of solutions, the preference information between two solutions is represented by linguistic 2-tuple.

Definition 5 [14] Let $\ddot{P}=\left(\ddot{p}_{i j}, \alpha_{i j}\right)_{n \times n}$ be a linguistic 2-tuple comparison matrix and the element $\left(\ddot{p}_{i j}, \alpha_{i j}\right)$ represent the result of comparing two solutions. If the following propositions are right,

(1) $\ddot{p}_{i j} \in S ; \quad \alpha_{i j} \in[-0.5,0.5]$;

(2) $\nabla^{-1}\left(\ddot{p}_{i i}, \alpha_{i i}\right)=g / 2$;

(3) $\nabla^{-1}\left(\ddot{p}_{i j}, \alpha_{i j}\right)+\nabla^{-1}\left(\ddot{p}_{j i}, \alpha_{j i}\right)=g$

then $\ddot{P}$ is called a linguistic 2-tuple judgment matrix. Proposition $\quad \nabla^{-1}\left(\ddot{p}_{i j}, \alpha_{i j}\right)+\nabla^{-1}\left(\ddot{p}_{j i}, \alpha_{j i}\right)=g$ means the linguistic 2-tuple judgment matrix is complementary. in another word, a linguistic 2-tuple judgment matrix is a complementary judgment matrix.

The element $\left(\ddot{p}_{i j}, \alpha_{i j}\right)$ in $\ddot{P}$ implies :

If $\nabla^{-1}\left(\ddot{p}_{i j}, \alpha_{i j}\right)=g / 2$ then the preference of the solution $\mathrm{x}_{\mathrm{i}}$ is same as the preference of the solution $\mathrm{x}_{\mathrm{j}}$. If $\nabla^{-1}\left(\ddot{p}_{i j}, \alpha_{i j}\right)<\mathrm{g} / 2$ then the solution $\mathrm{x}_{\mathrm{j}}$ is better than the solution $\mathrm{x}_{\mathrm{i}}$, and the smaller the $\nabla^{-1}\left(\ddot{p}_{i j}, \alpha_{i j}\right)$ the better the solution $\mathrm{x}_{\mathrm{j}}$ comparing the solution $\mathrm{x}_{\mathrm{i}}$. If $\nabla^{-1}\left(\ddot{p}_{i j}, \alpha_{i j}\right)>\mathrm{g} / 2$ then the solution $\mathrm{x}_{\mathrm{i}}$ is better than the solution $\mathrm{x}_{\mathrm{j}}$, and the bigger the $\nabla^{-1}\left(\ddot{p}_{i j}, \alpha_{i j}\right)$ the better the solution $\mathrm{x}_{\mathrm{i}}$ comparing the solution $\mathrm{x}_{\mathrm{j}}$.

Definition 6 [14] Let $\ddot{P}=\left(\ddot{p}_{i j}, \alpha_{i j}\right)_{n \times n}$ be a linguistic 2-tuple judgment matrix, if $\forall i, j, k \in I$, elements in $\ddot{P}$ has the properties of the formula (8), then $\ddot{P}$ is called a linguistic 2-tuple judgment matrix with additive consistency.

$$
\begin{array}{r}
\nabla^{-1}\left(\ddot{p}_{i j}, \alpha_{i j}\right)+\nabla^{-1}\left(\ddot{p}_{j k}, \alpha_{j k}\right) \\
=\nabla^{-1}\left(\ddot{p}_{i k}, \alpha_{i k}\right)+g / 2
\end{array}
$$

\section{Properties of a linguistic 2-tuple judgment matrix with additive consistency}

Let $X=\left\{x_{1}, x_{2}, \cdots, x_{n}\right\}$ be the set of solutions, the preference information between two solutions is represented by linguistic 2-tuple. The relation matrix $\ddot{P}=\left(\ddot{p}_{i j}, \alpha_{i j}\right)_{n \times n}$ of the preference relation

$R: X \rightarrow X$ is a linguistic 2-tuple judgment matrix with additive consistency. Then the preference relation $R: X \rightarrow X$ is a partial order $(\mathrm{X}, \leqslant)$ because the following propositions are right.

Reflexive: $\quad \forall i \in I \subseteq[0, g]$

$\nabla^{-1}\left(\ddot{p}_{i i}, \alpha_{i i}\right)+\nabla^{-1}\left(\ddot{p}_{i i}, \alpha_{i i}\right)=g$. Therefore, $\nabla^{-1}\left(\ddot{p}_{i i}, \alpha_{i i}\right)=g / 2$, that means every solution has the same preference.

Anti-symmetric: $\quad \forall i, j \in I \subseteq[0, g] \quad, \quad$ if $\left(x_{i}, x_{j}\right) \in R,\left(x_{j}, x_{i}\right) \in R \quad$, means $\nabla^{-1}\left(\ddot{p}_{i j}, \alpha_{i j}\right) \leq g / 2$ and $\nabla^{-1}\left(\ddot{p}_{j i}, \alpha_{j i}\right) \leq g / 2$. Thus, $\nabla^{-1}\left(\ddot{p}_{i j}, \alpha_{i j}\right)=g / 2$.

Transitive: $\quad \forall i, j, k \in I \subseteq[0, g] \quad, \quad$ if $\left(x_{i}, x_{j}\right) \in R,\left(x_{j}, x_{k}\right) \in R \quad$, means $\nabla^{-1}\left(\ddot{p}_{i j}, \alpha_{i j}\right) \leq g / 2$ and $\nabla^{-1}\left(\ddot{p}_{j k}, \alpha_{j k}\right) \leq g / 2$. Because $\nabla^{-1}\left(\ddot{p}_{i j}, \alpha_{i j}\right)+\nabla^{-1}\left(\ddot{p}_{j k}, \alpha_{j k}\right)$ $=\nabla^{-1}\left(\ddot{p}_{i k}, \alpha_{i k}\right)+g / 2, \forall i, j, k \in I$. We get $\nabla^{-1}\left(\ddot{p}_{i k}, \alpha_{i k}\right) \leq g / 2$. So $\left(x_{i}, x_{k}\right) \in R$.

From the definition of a linguistic 2-tuple judgment matrix with additive consistency and its 
related preference partial order relations, we can obtain the following properties.

Theorem 1 A linguistic 2-tuple judgment matrix with additive consistency $\ddot{P}$ can be obtained from $\ddot{P}_{0}=\left(\left(\ddot{p}_{12}, \alpha_{12}\right),\left(\ddot{p}_{23}, \alpha_{23}\right), \cdots,\left(\ddot{p}_{(n-1) n}, \alpha_{(n-1) n}\right)\right)$

Proof : From $\quad\left(\ddot{p}_{i(i+1)}, \alpha_{i(i+1)}\right) \quad$ and $\left(\ddot{p}_{(i+1)(i+2)}, \alpha_{(i+1)(i+2)}\right) \quad$, we get $\nabla^{-1}\left(\ddot{p}_{i(i+1)}, \alpha_{i(i+1)}\right)+\nabla^{-1}\left(\ddot{p}_{(i+1)(i+2)}, \alpha_{(i+1)(i+2)}\right)$ $=\nabla^{-1}\left(\ddot{p}_{i(i+2)}, \alpha_{i(i+2)}\right)+g / 2, i \leq n-2$. Then we get $\quad\left(\ddot{p}_{i(i+2)}, \alpha_{i(i+2)}\right)=\nabla\left(\beta_{i(i+2)}\right) \quad$, with $\beta_{i(i+2)}=\nabla^{-1}\left(\ddot{p}_{i(i+2)}, \alpha_{i(i+2)}\right)=\nabla^{-1}\left(\ddot{p}_{i(i+1)}, \alpha_{i(i+1)}\right)+$ $\nabla^{-1}\left(\ddot{p}_{(i+1)(i+2)}, \alpha_{(i+1)(i+2)}\right)-g / 2 \quad$. From $\left(\ddot{p}_{i(i+2)}, \alpha_{i(i+2)}\right)$ and $\left(\ddot{p}_{(i+2)(i+3)}, \alpha_{(i+2)(i+3)}\right)$ we get $\left(\ddot{p}_{i(i+3)}, \alpha_{i(i+3)}\right)$. Using mathematical induction we get element $\left(\ddot{p}_{i j}, \alpha_{i j}\right), i<j$. From $\left(\ddot{p}_{i j}, \alpha_{i j}\right)$ we can get $\left(\ddot{p}_{j i}, \alpha_{j i}\right)$ because a linguistic 2-tuple judgment matrix is complementary. Therefore, we can say

$\ddot{P}_{0}=\left(\left(\ddot{p}_{12}, \alpha_{12}\right),\left(\ddot{p}_{23}, \alpha_{23}\right), \cdots,\left(\ddot{p}_{(n-1) n}, \alpha_{(n-1) n}\right)\right)$ can built every element in a linguistic 2-tuple judgment matrix with additive consistency $\ddot{P}$.

Theorem 2 A linguistic 2-tuple judgment matrix $\ddot{P}$ is additive consistent $\Leftrightarrow$ There exists a vector $\beta=\left(\beta_{1}, \beta_{2}, \cdots, \beta_{n}\right) \quad$ which will make $\nabla^{-1}\left(\ddot{p}_{i j}, \alpha_{i j}\right)=\beta_{i}-\beta_{j}+g / 2$ true.

Proof: If a linguistic 2-tuple judgment matrix $\ddot{P}$ is additive consistent, according to theorem 2 the vector

$\ddot{P}_{0}=\left(\left(\ddot{p}_{12}, \alpha_{12}\right),\left(\ddot{p}_{23}, \alpha_{23}\right), \cdots,\left(\ddot{p}_{(n-1) n}, \alpha_{(n-1) n}\right)\right)$ can built every element in $\ddot{P}$. $\beta=\left(\beta_{1}, \beta_{2}, \cdots, \beta_{n}\right) \quad$ can be found to let $\nabla^{-1}\left(p_{i(i+1)}, \alpha_{i(i+1)}\right)=\beta_{i}-\beta_{(i+1)}+g / 2$ be true. Using mathematical induction we can get $\beta_{i}=\sum_{j=i}^{n-1} \nabla^{-1}\left(\ddot{p}_{j(j+1)}, \alpha_{j(j+1)}\right)+\beta_{n}-\frac{g(n-i)}{2}$. Thus, every element $\left(\ddot{p}_{i j}, \partial_{i j}\right)$ in $\ddot{P}$, $\nabla^{-1}\left(\ddot{p}_{i j}, \alpha_{i j}\right)=\beta_{i}-\beta_{j}+g / 2$ must be true.
If there exists a vector $\beta=\left(\beta_{1}, \beta_{2}, \cdots, \beta_{n}\right)$ which make $\nabla^{-1}\left(\ddot{p}_{i j}, \alpha_{i j}\right)=\beta_{i}-\beta_{j}+g / 2$ be true, then $\quad \nabla^{-1}\left(\ddot{p}_{i j}, \alpha_{i j}\right)+\nabla^{-1}\left(\ddot{p}_{j k}, \alpha_{j k}\right)$ $=\nabla^{-1}\left(\ddot{p}_{i k}, \alpha_{i k}\right)+g / 2$ for $\forall i, j, k \in I$ must be true. Therefore, the linguistic 2-tuple judgment matrix is additive consistent.

Theorem 3 If a linguistic 2-tuple judgment matrix $\ddot{P}$ is additive consistent then the following formula is true.

$\nabla^{-1}\left(\ddot{p}_{i j}, \alpha_{i j}\right)=$

$\sum_{k=i}^{j-1} \nabla^{-1}\left(\ddot{p}_{k(k+1)}, \alpha_{k(k+1)}\right)+\frac{g(i-j+1)}{2}$ for $i<j$;

$\nabla^{-1}\left(\ddot{p}_{i j}, \alpha_{i j}\right)=$

$-\sum_{k=j}^{i-1} \nabla^{-1}\left(\ddot{p}_{k(k+1)}, \alpha_{k(k+1)}\right)+\frac{g(i-j+1)}{2}$ for $i>j$.

Proof : According to theorem 2 , $\nabla^{-1}\left(\ddot{p}_{i j}, \alpha_{i j}\right)=\beta_{i}-\beta_{j}+g / 2 \quad$ and $\beta_{i}=\sum_{j=i}^{n-1} \nabla^{-1}\left(\ddot{p}_{j(j+1)}, \alpha_{j(j+1)}\right)+\beta_{n}-\frac{g(n-i)}{2}$ must be true. And $\nabla^{-1}\left(\ddot{p}_{i j}, \alpha_{i j}\right)=\sum_{k=i}^{n-1} \nabla^{-1}\left(\ddot{p}_{k(k+1)}, \alpha_{k(k+1)}\right)-$ $\sum_{k=j}^{n-1} \nabla^{-1}\left(\ddot{p}_{k(k+1)}, \alpha_{k(k+1)}\right)+\frac{g(i-j+1)}{2}$ is obtained. If $\quad \mathrm{i}<\mathrm{j}, \quad$ then $\quad \nabla^{-1}\left(\ddot{p}_{i j}, \alpha_{i j}\right)=$ $\sum_{k=i}^{j-1} \nabla^{-1}\left(\ddot{p}_{k(k+1)}, \alpha_{k(k+1)}\right)+\frac{g(i-j+1)}{2}$.

If $\mathrm{i} \quad>\mathrm{j}$, then $\nabla^{-1}\left(\ddot{p}_{i j}, \alpha_{i j}\right)=$ $-\sum_{k=j}^{i-1} \nabla^{-1}\left(\ddot{p}_{k(k+1)}, \alpha_{k(k+1)}\right)+\frac{g(i-j+1)}{2}$.

Theorem 4 A linguistic 2-tuple judgment matrix $\ddot{P}$ is additive consistent if and only if $\forall i, j \in I$, $\nabla^{-1}\left(\ddot{p}_{i k}, \alpha_{i k}\right)-\nabla^{-1}\left(\ddot{p}_{j k}, \alpha_{j k}\right)=c, k=1,2, \cdots, n$, $\mathrm{c}$ is a constant.

Proof: If linguistic 2-tuple judgment matrix $\ddot{P}$ is additive consistent, then $\nabla^{-1}\left(\ddot{p}_{i j}, \alpha_{i j}\right)=\beta_{i}-\beta_{j}+g / 2$ is true according to theorem $2 . \quad$ Therefore, $\nabla^{-1}\left(\ddot{p}_{i k}, \alpha_{i k}\right)-\nabla^{-1}\left(\ddot{p}_{j k}, \alpha_{j k}\right)=\beta_{i}-\beta_{j}=c$ 


$$
\begin{aligned}
& \text { Let } c=\nabla^{-1}\left(\ddot{p}_{i j}, \alpha_{i j}\right)-g / 2 \text { be true. If } \\
& \nabla^{-1}\left(\ddot{p}_{i k}, \alpha_{i k}\right)-\nabla^{-1}\left(\ddot{p}_{j k}, \alpha_{j k}\right)=c, k=1,2, \cdots, n \\
& \text { be true, then } \nabla^{-1}\left(\ddot{p}_{i j}, \alpha_{i j}\right)+\nabla^{-1}\left(\ddot{p}_{j k}, \alpha_{j k}\right) \\
& =\nabla^{-1}\left(\ddot{p}_{i k}, \alpha_{i k}\right)+g / 2 \text { for } \forall i, j, k \in I .
\end{aligned}
$$

\section{Conclusions}

This paper studies the additive consistency of a linguistic 2-tuple judgment matrix according to the definitions of a linguistic 2-tuple judgment matrix and its additive consistency. It puts forward several propositions about the properties of a linguistic 2-tuple judgment matrix with additive consistency. The paper also proves them. With the help of these propositions, it will be helpful to judge whether a linguistic 2-tuple judgment matrix is additive consistent or not. These properties can be used as a tool to build a linguistic 2tuple judgment matrix with additive consistency and to sort the solutions based on linguistic 2-tuple judgment matrix with additive consistency. Meanwhile, these propositions will be a basis for aggregation analysis of group decision making based on linguistic 2-tuple.

The additive consistency of a linguistic 2-tuple judgment matrix will be further studied.

\section{References}

[1] D. Ben-Arieh, Z. Chen, Linguisitic group decision-making: opinion aggregation and measures of consensus. Fuzzy Optim Decis Making, 5:371-386, 2006.

[2] J. Ye, Y.C. Xu, A decision making method based on similarity measure of vague sets. Chinese journal of computer simulation, 24(4):90-93, 2006 (in Chinese).

[3] Q. Mou, Q.J. Wu, C.G. Lu, A Multiple Indices Approach for Ranking Fuzzy Number. Journal of Guangxi Teachers Education University (Natural Science Edition), 21(2):17-21 , 2006 (in Chinese).

[4] Z.S. Xu, A multi-attribute group decision making method based on term indexes in linguistic scale. Journal of System engineering, 20(1):84-88, 2005 (in Chinese).

[5] F. Herrera, L. Nartinez, A 2-tuple fuzzy linguistic representation model for computing with words. IEEE Transaction on Fuzzy Systems, 8(6):746-752, 2000.

[6] Z.S. Xu, A Multi-attribute group decision making method based on fuzzy linguistic evaluationa and GIOWA operator. J.Sys.Sci. \& Math. Sci., 24(2):218-224, 2004 (in Chinese).
[7] F. Herrera, L. Martinez, A model based on linguistic 2-tuples for dealing with multigranular hierarchical linguistic contexts in multi-expert decision making. IEEE Transactions on Systems, Man, and Cybernetics-part b: Cybernetics, 31(2):227-234, 2001.

[8] F. Herrera, L. Martinez, The 2-tuple linguistic computational model-advantages of its linguistic description, accuracy and consistency. International Journal of Uncertainty, Fuzziness and Knowledge-Based Systems, 9:33-48, 2003.

[9] M. Delgado, F. Herrera, E. Herrera-Viedma, M.J. Martin-Bautista, L. Martinez, M.A. Vila, A communication model based on the 2-tuple fuzzy linguistic representation for a distributed intelligent agent system on internet. Soft Computing, 6:320-328, 2006.

[10] X.R. Wang, Z.P. Fan, Method for group decision making based on two-tuple linguistic information processing. Journal of management sciences in China, 6(5):1-5, 2003 (in Chinese).

[11] H.Y. Li, Z.P. Fan, A multi-attribute group decision making method based on linguistic 2tuple. Journal of Northeastern University (Natural Science), 24(5):495-498, 2005.

[12] C.H. Yu, Z.P. Fan, Maximal tree clustering method based on dyadic semantic information processing. Systems Engineering and Electronics, 28(10):1519-1522, 2006 (in Chinese).

[13] X.W. Liao, H. Li, G.M. Dong, A Multi-attribute Group Decision-making Approach Dealing with Linguistic Assessment Information. Theory and Practice of System Engineering, 9:90-98, 2006 (in Chinese).

[14] Y.P. Jiang, Y.N. Xing, Consistency Analysis of Two-Tuple Linguistic Judgment Matrix. Journal of Northeastern University (Natural Science), 28(1):129-132, 2007.

[15] W.X. Zhang, G.F. Qiu, Uncertainty decision making based on rough sets, Tsinghua University Publisher, Beijing, 2005 (in Chinese).

[16] Y. Chen, Z.P. Fan, Consistency and its related application research on linguistic judgment matrix. Theory and Practice of System Engineering, 24(4):136-141, 2004 (in Chinese).

[17] Y.J. Lv, X.R. Guo, G.L. Xu, A new way for improving consistency of the fuzzy complementary judgment matrices. Journal of Guangxi University (Nat Sci Ed), 31(4):281-287, 2006. 